\title{
Finite-Time Synchronization of Singular Hybrid Coupled Networks
}

\author{
Cong Zheng' and Jinde Cao ${ }^{1,2}$ \\ ${ }^{1}$ Research Center for Complex Systems and Network Sciences, and Department of Mathematics, Southeast University, \\ Nanjing 210096, China \\ ${ }^{2}$ Department of Mathematics, King Abdulaziz University, Jeddah 21589, Saudi Arabia
}

Correspondence should be addressed to Jinde Cao; jdcao@seu.edu.cn

Received 20 December 2012; Accepted 11 February 2013

Academic Editor: Jong Hae Kim

Copyright (C) 2013 C. Zheng and J. Cao. This is an open access article distributed under the Creative Commons Attribution License, which permits unrestricted use, distribution, and reproduction in any medium, provided the original work is properly cited.

\begin{abstract}
This paper investigates finite-time synchronization of the singular hybrid coupled networks. The singular systems studied in this paper are assumed to be regular and impulse-free. Some sufficient conditions are derived to ensure finite-time synchronization of the singular hybrid coupled networks under a state feedback controller by using finite-time stability theory. A numerical example is finally exploited to show the effectiveness of the obtained results.
\end{abstract}

\section{Introduction}

In recent years, singular systems, also known as descriptor systems, generalized state-space systems, differentialalgebraic systems, or semistate systems, are attracting more and more attentions from many fields of scientific research because they can better describe a larger class of dynamic systems than the regular ones. Many results of regular systems have been extended to the area about singular systems such as [1-21]. For example, stability (robust stability or quadratic stability) and stabilization for singular systems have been studied via LMI approach in [2-8]; robust control (or $\mathrm{H}_{2}$, $H_{\infty}$ control and robust dissipative filtering) for singular systems has been discussed in [9-16]; synchronization (or state estimation) for singular complex networks has been considered in [17-21].

Synchronization is an interesting and important characteristic in the coupled networks. There are a lot of results in regular coupled networks. Recently, some authors study synchronization of the singular systems such as [17-21] and the references therein. In [17], Xiong et al. introduced the singular hybrid coupled systems to describe complex network with a special class of constrains. They gave a sufficient condition for global synchronization of singular hybrid coupled system with time-varying nonlinear perturbation based on Lyapunov stability theory. Synchronization issues are studied for singular systems with delays by using Linear Matrix Inequality (LMI) approach [18]. Koo et al. considered synchronization of singular complex dynamical network with time-varying delays [19]. Li et al. in [20] investigated synchronization and state estimation for singular complex dynamical networks with time-varying delays. $\mathrm{Li}$ et al. in [21] investigated robust $H_{\infty}$ control of synchronization for uncertain singular complex delayed networks with stochastic switched coupling.

Finite-time synchronization or finite-time control is interesting topic for its practical application. There are some results on finite-time stability [22-26], finite-time synchronization [27-33], finite-time consensus or agreement [3437], and finite-time observers [38]. However, these results are obtained for regular systems. Up to now, to the best of our knowledge, few authors studied finite-time synchronization of singular hybrid coupled systems whose structures are more complex than those in [27-33]. Considering the important role of synchronization of complex networks, the finite-time synchronization of singular hybrid coupled networks is worth studying.

Motivated by the previous discussions, in this paper, we investigate finite-time synchronization of singular hybrid complex systems. Some sufficient conditions for it are 
obtained by the state feedback controller based on the finitetime stability theory. Finally, a numerical example is exploited to illustrate the effectiveness of the obtained result.

The rest of this paper is organized as follows. In Section 2, a singular hybrid coupled system is given, and some preliminaries are briefly outlined. In Section 3, some sufficient criteria are derived for the finite-time synchronization of the proposed singular system by the feedback controller. In Section 4, an example is provided to show the effectiveness of the obtained results. Some conclusions are finally drawn in Section 5 .

\section{Model Formulation and Some Preliminaries}

Consider a singular hybrid coupled system as follows:

$$
\begin{array}{r}
E \dot{x}_{i}(t)=A x_{i}(t)+f\left(x_{i}(t), t\right)+c \sum_{j=1}^{N} b_{i j}(t) \Gamma x_{j}(t), \\
i=1,2, \ldots, N,
\end{array}
$$

where $x_{i}(t)=\left(x_{i 1}(t), x_{i 2}(t), \ldots, x_{i n}(t)\right)^{T} \in \mathbb{R}^{n}$ represents the state vector of the $i$ th node, $A, E \in \mathbb{R}^{n \times n}$ are constant matrices, and $E$ may be singular. Without loss of generality, we will assume that $0<\operatorname{rank}(E)=r<n . f\left(x_{i}(t), t\right)$ is a vector-value function. The constant $c>0$ denotes the coupling strength, and $\Gamma=\operatorname{diag}\left(\gamma_{1}, \gamma_{2}, \ldots, \gamma_{n}\right) \in \mathbb{R}^{n \times n}$ is inner-coupling matrix between nodes. $B=\left(b_{i j}\right)_{N \times N}$ describes the linear coupling configuration of the network, which satisfies

$$
\begin{gathered}
b_{i j}=b_{j i}, \quad \text { for } i \neq j, \\
b_{i i}=-\sum_{j=1, j \neq i}^{N} b_{i j}, \quad i=1,2, \ldots, N .
\end{gathered}
$$

Remark 1. If $\operatorname{rank}(E)=n$, then system (1) is a general nonsingular coupled network. We will also give a sufficient condition of the finite-time synchronization for this circumstance. See Corollary 10.

Definition 2. The singular system (1) is said to be synchronized in the finite time, if for a suitable designed feedback controller, there exists a constant $t^{*}>0$ (which depends on the initial vector value $\left.x(0)=\left(x_{1}^{T}(0), x_{2}^{T}(0), \ldots, x_{N}^{T}(0)\right)^{T}\right)$, such that $\lim _{t \rightarrow t^{*}}\left\|x_{i}(t)-x_{j}(t)\right\|=0$ and $\left\|x_{i}(t)-x_{j}(t)\right\| \equiv 0$ for $t>t^{*}, i, j=1,2, \ldots, N$.

Assumption 3. Assume that the singular system (1) is connected in the sense that there are no isolated clusters; that is, the matrix $B$ is an irreducible matrix.

With Assumption 3, we obtain that zero is an eigenvalue of $B$ with multiplicity 1 , and all the other eigenvalues of $B$ are strictly negative, which are denoted by $0=\lambda_{1}>$ $\lambda_{2} \geq \cdots \geq \lambda_{N}$. At the same time, since $B$ is a symmetric matrix, there exists a unitary matrix $W=\left(W_{1}, W_{2}, \ldots, W_{N}\right) \in$ $\mathbb{R}^{n \times n}$ such that $B=W \Lambda W^{T}$ with $W W^{T}=I$ and $\Lambda=$ $\operatorname{diag}\left(\lambda_{1}, \lambda_{2}, \ldots, \lambda_{N}\right)$.
Let $s(t)$ be a function to which all $x_{i}(t)$ are expected to synchronize in the finite time. That is, the synchronization state is $s(t)$. Suppose that $s(t)$ satisfies the equation $E \dot{s}(t)=$ $A s(t)+f(s(t), t)$. Let $e_{i}(t)=x_{i}(t)-s(t), i=1,2, \ldots, N$. We can obtain the following singular error system:

$$
\begin{aligned}
E \dot{e}_{i}(t)= & A e_{i}(t)+f\left(x_{i}(t), t\right)-f(s(t), t) \\
& +c \sum_{j=1}^{N} b_{i j}(t) \Gamma e_{j}(t), \quad i=1,2, \ldots, N .
\end{aligned}
$$

Let $e(t)=\left(e_{1}(t), e_{2}(t), \ldots, e_{N}(t)\right), y(t)=e(t) W$; then system (3) can be written as

$$
\begin{aligned}
& E \dot{e}(t)=A e(t)+F(e(t), t)+c \Gamma e(t) B^{T}, \\
& E \dot{y}(t)=A y(t)+F(e(t), t) W+c \Gamma y(t) \Lambda,
\end{aligned}
$$

where $F(e(t), t)=\left(f\left(x_{1}(t), t\right)-f(s(t), t), f\left(x_{2}(t), t\right)-\right.$ $\left.f(s(t), t), \ldots, f\left(x_{N}(t), t\right)-f(s(t), t)\right), y(t)=\left(y_{1}(t), y_{2}(t), \ldots\right.$, $\left.y_{N}(t)\right)$, and $y_{i}(t)=e(t) W_{i} \in \mathbb{R}^{n}, i=1,2, \ldots, N$. Then, system (4) can be written as

$$
\begin{aligned}
E \dot{y}_{i}(t) & =A y_{i}(t)+F(e(t), t) W_{i}+c \lambda_{i} \Gamma y_{i}(t) \\
& =\left(A+c \lambda_{i} \Gamma\right) y_{i}(t)+F(e(t), t) W_{i} .
\end{aligned}
$$

Therefore, the finite-time synchronization problem of system (1) is equivalent to the finite-time stabilization of system (5) at the origin under the suitable controllers $u_{i}$, $i=1,2, \ldots, N$.

Assumption 4. Assume that there exist nonnegative constants $L_{i}$ such that

$$
\begin{array}{r}
\left\|f\left(x_{i}(t), t\right)-f(s(t), t)\right\| \leq L_{i}\left\|x_{i}(t)-s(t)\right\|, \\
i=1,2, \ldots, N .
\end{array}
$$

Assumption 5. There exist matrices $P_{i}$ such that

$$
\begin{gathered}
E^{T} P_{i}=P_{i}^{T} E \geq 0, \quad i=1,2, \ldots, N, \\
A^{T} P_{1}+P_{1}^{T} A<0, \\
\left(A+c \lambda_{i} \Gamma\right)^{T} P_{i}+P_{i}^{T}\left(A+c \lambda_{i} \Gamma\right) \leq-\eta_{i} I, \quad i=2, \ldots, N,
\end{gathered}
$$

where $\eta_{i}>2 L(N-1)\left\|P_{i}\right\|, L=\sum_{i=1}^{N} L_{i}$.

Lemma 6 (see [26]). Suppose that the function $V(t)$ : $\left[t_{0}\right.$, $\infty) \rightarrow[0, \infty)$ is differentiable (the derivative of $V(t)$ at $t_{0}$ is in fact its right derivative) and $\dot{V}(t) \leq-K(V(t))^{\alpha}, \forall t \geq 0$, $V\left(t_{0}\right) \geq 0$, where $K>0,0<\alpha<1$ are two constants. Then, for any given $t_{0}, V(t)$ satisfies the following inequality:

$$
\begin{gathered}
V^{1-\alpha}(t) \leq V^{1-\alpha}\left(t_{0}\right)-K(1-\alpha)\left(t-t_{0}\right), \quad t_{0} \leq t \leq t^{*}, \\
V(t) \equiv 0, \quad \forall t>t^{*},
\end{gathered}
$$

with $t^{*}$ given by $t^{*}=t_{0}+V^{1-\alpha}\left(t_{0}\right) / K(1-\alpha)$. 
Lemma 7 (Jensen's Inequality). If $a_{1}, a_{2}, \ldots, a_{n}$ are positive numbers and $0<r<p$, then

$$
\left(\sum_{i=1}^{n} a_{i}^{p}\right)^{(1 / p)} \leq\left(\sum_{i=1}^{n} a_{i}^{r}\right)^{(1 / r)} .
$$

\section{Main Results}

In this section, we consider the finite-time synchronization of the singular coupled network (1) under the appropriate controllers. In order to control the states of all nodes to the synchronization state $s(t)$ in finite time, we apply some simple controllers $u_{i}(t) \in \mathbb{R}^{n}, i=1,2, \ldots, N$, to system (1). Then, the controlled system can be written as

$$
\begin{array}{r}
E \dot{x}_{i}(t)=A x_{i}(t)+f\left(x_{i}(t), t\right)+c \sum_{j=1}^{N} b_{i j} \Gamma x_{j}(t)+u_{i}, \\
i=1,2, \ldots, N .
\end{array}
$$

Then, we have

$$
\begin{aligned}
& E \dot{e}_{i}(t)= A e_{i}(t)+f\left(x_{i}(t), t\right)-f(s(t), t) \\
&+c \sum_{j=1}^{N} b_{i j} \Gamma e_{j}(t)+u_{i}, \\
& E \dot{y}_{i}(t)=\left(A+c \lambda_{i} \Gamma\right) y_{i}(t)+F(e(t), t) W_{i}+v_{i},
\end{aligned}
$$

where $v_{i}=u W_{i}, u=\left(u_{1}, u_{2}, \ldots, u_{N}\right)$.

With Assumption 5, it follows from the proof of Theorem 1 in [2] and Lemma 2.2 in [3] that the pair $\left(E, A+c \lambda_{i} \Gamma\right)$ is regular and impulse-free; that is, there exist nonsingular matrices $M_{i}, Q_{i} \in \mathbb{R}^{n \times n}$ satisfying that

$$
\begin{gathered}
M_{i} E Q_{i}=\operatorname{diag}\left\{I_{r}, 0\right\}, \\
M_{i}\left(A+c \lambda_{i} \Gamma\right) Q_{i}=\operatorname{diag}\left\{A_{i}, I_{n-r}\right\},
\end{gathered}
$$

where $A_{i} \in \mathbb{R}^{r \times r}, i=1,2, \ldots, N$. So, system (13) is equivalent to

$$
\begin{gathered}
\dot{y}_{i}^{1}(t)=A_{i} y_{i}^{1}(t)+M_{i}^{1} F(e(t), t) W_{i}+M_{i}^{1} v_{i}, \\
0=y_{i}^{2}(t)+M_{i}^{2} F(e(t), t) W_{i}+M_{i}^{2} v_{i},
\end{gathered}
$$

where $Q_{i}^{-1} y_{i}(t)=\left(\begin{array}{l}y_{i}^{1}(t) \\ y_{i}^{2}(t)\end{array}\right), y_{i}^{1}(t) \in \mathbb{R}^{r}$, and $y_{i}^{2}(t) \in \mathbb{R}^{n-r}$. And $M_{i}=\left(\begin{array}{l}M_{i}^{1} \\ M_{i}^{2}\end{array}\right), M_{i}^{1} \in \mathbb{R}^{r \times n}, M_{i}^{2} \in \mathbb{R}^{(n-r) \times n}, Q_{i}=\left(Q_{i}^{1} Q_{i}^{2}\right), Q_{i}^{1} \in$ $\mathbb{R}^{n \times r}$, and $Q_{i}^{2} \in \mathbb{R}^{n \times(n-r)}$

In order to achieve our aim, we design the following controllers:

$$
v_{i}=-k M_{i}^{-1} \operatorname{sign}\left(M_{i} E e(t) W_{i}\right)\left|M_{i} E e(t) W_{i}\right|^{\beta},
$$

where

$$
\begin{aligned}
& M_{i} E e(t) W_{i}=M_{i} E y_{i}(t)=M_{i} E Q_{i} Q_{i}^{-1} y_{i}(t) \\
& =\left(\begin{array}{cc}
I_{r} & 0 \\
0 & 0
\end{array}\right)\left(\begin{array}{c}
y_{i}^{1} \\
y_{i}^{2}
\end{array}\right)=\left(\begin{array}{c}
y_{i}^{1} \\
0
\end{array}\right), \\
& \left|M_{i} E y_{i}(t)\right|^{\beta}=(\left|y_{i 1}^{1}(t)\right|^{\beta}, \ldots,\left|y_{i r}^{1}(t)\right|^{\beta}, \underbrace{0, \ldots, 0}_{n-r})^{T}, \\
& \operatorname{sign}\left(M_{i} E y_{i}(t)\right)=\operatorname{diag}\left(\operatorname{sign}\left(y_{i 1}^{1}(t)\right), \operatorname{sign}\left(y_{i 2}^{1}(t)\right), \ldots,\right. \\
& \quad \operatorname{sign}\left(y_{i r}^{1}(t)\right), \underbrace{0, \ldots, 0}_{n-r}) .
\end{aligned}
$$

$k>0$ is a tunable constant, and the real number $\beta$ satisfies $0<\beta<1$. So, we obtain $u=\left(v_{1}, v_{2}, \ldots, v_{N}\right) W^{-1}=$ $v\left(\xi_{1}, \xi_{2}, \ldots, \xi_{N}\right)$. That is, $u_{i}=v \xi_{i}$.

Remark 8. From (17), the controllers $u_{i}$ are dependent not only on the coupled matrix $B$, but also on the singular matrix $E$. And from the shape of controllers, we only use the states $y_{i}^{1}$ of slow subsystems (15) in controllers $v_{i}$, but we do not consider the states $y_{i}^{2}$ of fast subsystems (16). It is very special. It is interesting for our future research to design more general controller which makes the singular hybrid coupled networks synchronize in finite time.

Theorem 9. Suppose that Assumptions 3, 4, and 5 hold. Under the controllers (17), the singular system (1) is synchronized in a finite time $t^{*}=t_{0}+\left(V^{(1-\beta) / 2}\left(t_{0}\right) /\right.$ $\left.a k b^{-(1+\beta) / 2}(1-\beta)\right)$, where $V\left(t_{0}\right)=\sum_{i=1}^{N} y_{i}^{T}\left(t_{0}\right) E^{T} P_{i} y_{i}\left(t_{0}\right)=$ $\sum_{i=1}^{N}\left(e\left(t_{0}\right) W_{i}\right)^{T} E^{T} P_{i}\left(e\left(t_{0}\right) W_{i}\right), e\left(t_{0}\right)$ is the initial condition of $e(t)$, and $a$ and $b$ are defined as (25).

Proof. Consider the following Lyapunov function:

$$
V(t)=\sum_{i=1}^{N} y_{i}^{T}(t) E^{T} P_{i} y_{i}(t) .
$$

The derivative of $V(t)$ along the trajectory of system (13) is $\dot{V}(t)$

$$
\begin{gathered}
=\sum_{i=1}^{N}\left[y_{i}^{T}(t) P_{i}^{T}\left(\left(A+c \lambda_{i} \Gamma\right) y_{i}(t)+F(e(t), t) W_{i}+v_{i}\right)\right. \\
\left.\quad+\left(\left(A+c \lambda_{i} \Gamma\right) y_{i}(t)+F(e(t), t) W_{i}+v_{i}\right)^{T} P_{i} y_{i}(t)\right] \\
=\sum_{i=1}^{N}\left[y_{i}^{T}(t)\left(P_{i}^{T}\left(A+c \lambda_{i} \Gamma\right)+\left(A+c \lambda_{i} \Gamma\right)^{T} P_{i}\right) y_{i}(t)\right. \\
+2 y_{i}^{T}(t) P_{i}^{T} F(e(t), t) W_{i} \\
\left.\quad-2 k y_{i}^{T}(t) P_{i}^{T} M_{i}^{-1} \operatorname{sign}\left(M_{i} E e(t) W_{i}\right)\left|M_{i} E e(t) W_{i}\right|^{\beta}\right] .
\end{gathered}
$$


By using Assumption 4, one can get the following inequality:

$$
\begin{aligned}
\left\|F(e(t), t) W_{i}\right\| & =\left\|\sum_{k=1}^{N}\left[f\left(x_{k}(t), t\right)-f(s(t), t)\right] W_{i k}\right\| \\
& \leq \sum_{k=1}^{N} L_{k}\left\|e_{k}(t)\right\| \\
& =\sum_{k=1}^{N} L_{k}\left\|y(t) \xi_{k}\right\| \leq \sum_{k=1}^{N} L_{k}\|y(t)\|=L\|y(t)\| \\
& \leq L \sum_{k=1}^{N}\left\|y_{k}(t)\right\|,
\end{aligned}
$$

where $W_{i}=\left(W_{i 1}, W_{i 2}, \ldots, W_{i n}\right)^{T}$ and $\left(\xi_{1}, \xi_{2}, \ldots, \xi_{N}\right)=W^{-1}=$ $W^{T}$.

Define $M_{i}^{-T} P_{i} Q_{i}=\left(\begin{array}{cc}P_{i}^{1} & P_{i}^{2} \\ P_{i}^{3} & P_{i}^{4}\end{array}\right)$, where $P_{i}^{1} \in \mathbb{R}^{r \times r}, P_{i}^{2} \in$ $\mathbb{R}^{r \times(n-r)}, P_{i}^{3} \in \mathbb{R}^{(n-r) \times r}$, and $P_{i}^{4} \in \mathbb{R}^{(n-r) \times(n-r)}$. Using (7) and (8) (see [2]), one can obtain that $P_{i}^{1}=\left(P_{i}^{1}\right)^{T}>0$ and $P_{i}^{2}=0$; then,

$$
\begin{aligned}
& V(t)=\sum_{i=1}^{N} y_{i}^{T}(t) E^{T} P_{i} y_{i}(t)=\sum_{i=1}^{N}\left(y_{i}^{1}(t)\right)^{T} P_{i}^{1} y_{i}^{1}(t), \\
& -2 k y_{i}^{T}(t) P_{i}^{T} M_{i}^{-1} \operatorname{sign}\left(M_{i} E e(t) W_{i}\right)\left|M_{i} E e(t) W_{i}\right|^{\beta} \\
& =-2 k y_{i}^{T}(t) P_{i}^{T} M_{i}^{-1}\left(\operatorname{sign}\left(y_{i 1}^{1}(t)\right)\left|y_{i 1}^{1}(t)\right|^{\beta}, \ldots,\right. \\
& \left.\operatorname{sign}\left(y_{i r}^{1}(t)\right)\left|y_{i r}^{1}(t)\right|^{\beta}, 0, \ldots, 0\right) \\
& =-2 k y_{i}^{T}(t) Q_{i}^{-T}\left(\begin{array}{cc}
P_{i}^{1} & 0 \\
P_{i}^{3} & P_{i}^{4}
\end{array}\right)^{T} M_{i} M_{i}^{-1} \\
& \times \operatorname{diag}\left(\operatorname{sign}\left(y_{i}^{1}(t)\right), 0\right)\left(\begin{array}{c}
\left|y_{i}^{1}(t)\right|^{\beta} \\
0
\end{array}\right) \\
& =-2 k y_{i}^{1 T}(t) P_{i}^{1 T} \operatorname{sign}\left(y_{i}^{1}(t)\right)\left|y_{i}^{1}(t)\right|^{\beta} \\
& \leq-2 k \lambda_{\min }\left(P_{i}^{1 T}\right)\left|y_{i}^{1}(t)\right|^{1+\beta} \text {. }
\end{aligned}
$$

Substituting (8), (21), and (23) into (20) and letting $\eta_{1}=0$, while $\eta_{i} \geq 2 L(N-1)\left\|P_{i}\right\|, i=2, \ldots, N$, one has

$$
\begin{gathered}
\dot{V}(t) \leq \sum_{i=1}^{N}\left[-\eta_{i}\left\|y_{i}(t)\right\|^{2}+2 L\left\|P_{i}\right\| \sum_{j=1}^{N}\left\|y_{j}(t)\right\|\left\|y_{i}(t)\right\|\right. \\
\left.-2 k \lambda_{\min }\left(P_{i}^{1 T}\right)\left|y_{i}^{1}(t)\right|^{1+\beta}\right] \\
\leq \sum_{i=1}^{N}-2 k \lambda_{\min }\left(P_{i}^{1 T}\right)\left|y_{i}^{1}(t)\right|^{1+\beta}
\end{gathered}
$$

Let

$$
a \triangleq \min _{i}\left\{\lambda_{\min }\left(P_{i}^{1 T}\right)\right\}, \quad b \triangleq \max _{i}\left\{\lambda_{\max }\left(P_{i}^{1 T}\right)\right\} .
$$

From (22), we get

$$
a \sum_{i=1}^{N}\left\|y_{i}^{1}(t)\right\|^{2} \leq V(t) \leq b \sum_{i=1}^{N}\left\|y_{i}^{1}(t)\right\|^{2} .
$$

By the use of (24)-(26) and Lemma 7, we can obtain that

$$
\begin{aligned}
\dot{V}(t) & \leq-2 a k \sum_{i=1}^{N}\left|y_{i}^{1}(t)\right|^{1+\beta} \leq-2 a k\left(\sum_{i=1}^{N}\left\|y_{i}(t)\right\|^{2}\right)^{(1+\beta) / 2} \\
& \leq-2 a k\left(\frac{1}{b} V(t)\right)^{(1+\beta) / 2}=-2 a k b^{-(1+\beta) / 2}(V(t))^{(1+\beta) / 2} .
\end{aligned}
$$

From Lemma 6, we have that the solutions $y_{i}^{1}(t)$ of system (15) are globally asymptotically stable with respect to $y_{i}^{1}(t)=$ 0 in the finite time $t^{*}$; that is,

$$
\lim _{t \rightarrow t^{*}}\left\|y_{i}^{1}(t)\right\|=0, \quad\left\|y_{i}^{1}(t)\right\|=0 \quad \text { for } t \geq t^{*},
$$

where $t^{*}=t_{0}+\left(V^{(1-\beta) / 2}\left(t_{0}\right) / a k b^{-(1+\beta) / 2}(1-\beta)\right)$.

In the following, we show that $y_{i}^{2}(t)$ are globally asymptotically stable with respect to $y_{i}^{2}(t)=0$ in the finite time $t^{*}$. From (16), one has

$$
\left\|y_{i}^{2}(t)\right\| \leq\left\|M_{i}^{2}\right\|\left\|F(e(t), t) W_{i}\right\|+\left\|M_{i}^{2}\right\|\left\|v_{i}\right\| .
$$

Similar to the proof of Lemma 2.2 in [3] and the proof of Theorem 1 in [17], let $M_{i}^{2}\left(M_{i}^{2}\right)^{T}=I_{n-r}$, which implies that $\left\|M_{i}^{2}\right\|=1$. One has

$$
\begin{aligned}
\left\|y_{i}^{2}(t)\right\| \leq & \left\|F(e(t), t) W_{i}\right\|+\left\|u_{i} W_{i}\right\| \\
\leq & L \sum_{j=1}^{N}\left\|y_{j}(t)\right\|+\|-k M_{i}^{-1} \operatorname{sign}\left(M_{i} E y_{i}(t)\right) \\
& \times\left|M_{i} E y_{i}(t)\right|^{\beta} \| \\
\leq & L \sum_{j=1}^{N}\left\|Q_{j}\right\|\left(\left\|y_{j}^{1}(t)\right\|+\left\|y_{j}^{2}(t)\right\|\right)+k\left\|M_{i}^{-1}\right\| \\
& \times\left|y_{i}^{1}(t)\right|^{\beta} .
\end{aligned}
$$

Then,

$$
\begin{gathered}
\sum_{i=1}^{N}\left\|y_{i}^{2}(t)\right\| \leq \sum_{i=1}^{N}\left[L \sum_{j=1}^{N}\left\|Q_{j}\right\|\left(\left\|y_{j}^{1}(t)\right\|+\left\|y_{j}^{2}(t)\right\|\right)\right. \\
\left.+k\left\|M_{i}^{-1}\right\|\left|y_{i}^{1}(t)\right|^{\beta}\right] ;
\end{gathered}
$$


that is,

$$
\begin{aligned}
\sum_{i=1}^{N}\left(1-N L\left\|Q_{i}\right\|\right)\left\|y_{i}^{2}(t)\right\| \leq \sum_{i=1}^{N}( & N L\left\|Q_{i}\right\|\left\|y_{j}^{1}(t)\right\| \\
& \left.+k\left\|M_{i}^{-1}\right\|\left|y_{i}^{1}(t)\right|^{\beta}\right) .
\end{aligned}
$$

With Assumption 4, there must exist nonsingular matrices $M_{i}, Q_{i} \in \mathbb{R}^{n \times n}$ satisfying the equalities $M_{i} E Q_{i}=$ $\operatorname{diag}\left\{I_{r}, 0\right\}, M_{i}\left(A+c \lambda_{i} \Gamma\right) Q_{i}=\operatorname{diag}\left\{A_{i}, I_{n-r}\right\}$, where $A_{i} \in \mathbb{R}^{r \times r}$, $i=1,2, \ldots, N$. Moreover, nonsingular matrices $Q_{i}$ can be suitably chosen to satisfy $1-N L\left\|Q_{i}\right\|>0$, for $\forall i \in$ $\{1,2, \ldots, N\}$. Therefore, one can obtain $\lim _{t \rightarrow t^{*}}\left\|y_{i}^{2}(t)\right\|=0$, and $\left\|y_{i}^{2}(t)\right\|=0$ for $t \geq t^{*}$ from (28) and (32), $i=1,2, \ldots, N$. Consequently, $\lim _{t \rightarrow t^{*}}\left\|e_{i}(t)\right\|=0$, and $\left\|e_{i}(t)\right\|=0$ for $t \geq t^{*}, i=1,2, \ldots, N$. The proof is completed.

If $\operatorname{rank}(E)=n$, system (1) is a general nonsingular coupled network. By using the controllers $u_{i}$ similar to $v_{i}$ in (17), we can derive the finite-time synchronization of system (1). For simplicity, let $E=I_{n}$. Then, we have the following.

Corollary 10. When $E=I_{n}$, under Assumptions 3 and 4, let the controllers $u_{i}(t)$ be as follows:

$$
u_{i}(t)=-k \operatorname{sign}\left(e_{i}(t)\right)\left|e_{i}(t)\right|^{\beta}, \quad i=1,2, \ldots, N
$$

system (1) is synchronized in a finite time.

Remark 11. Since the conditions in Assumption 5 are not strict LMIs problems, they cannot be solved directly by the LMI Matlab Toolbox. According to Lemma 1 in [17], Lemma 1 in [9], and Remark 3 in [18], if matrix $E$ has the decomposition as

$$
E=U\left(\begin{array}{cc}
I_{r} & 0 \\
0 & 0
\end{array}\right)\left(\begin{array}{cc}
\Xi_{r} & 0 \\
0 & I_{n-r}
\end{array}\right) V^{T},
$$

where $U=\left(U_{1}, U_{2}\right), V=\left(V_{1}, V_{2}\right)$, and $\Xi_{r}=\operatorname{diag}\left\{\varrho_{1}, \ldots, \varrho_{r}\right\}$ with $\varrho_{i}>0$ for $i=1,2, \ldots, r$, then Assumption 5 can be transformed into a strict LMIs problem.

Corollary 12. Suppose that Assumptions 3 and 4 hold, and matrix $E$ has the decomposition as (34) in Remark 11. By the controllers (17), the singular hybrid coupled network (1) can be synchronized in the finite time in the sense of Definition 2, if there exist matrices $T_{i} \in \mathbb{R}^{r \times r}, T_{i} \geq 0$, and $S_{i} \in \mathbb{R}^{(n-r) \times r}$, $i=1,2, \ldots, N$, such that

$$
\begin{aligned}
& A^{T}\left(U_{1} T_{1} U_{1}^{T} E+U_{2} S_{1}\right)+\left(U_{1} T_{1} U_{1}^{T} E+U_{2} S_{1}\right)^{T} A<0, \\
& \left(A+c \lambda_{i} \Gamma\right)^{T}\left(U_{1} T_{i} U_{1}^{T} E+U_{2} S_{i}\right) \\
& \quad+\left(U_{1} T_{i} U_{1}^{T} E+U_{2} S_{i}\right)^{T}\left(A+c \lambda_{i} \Gamma\right) \leq-\eta_{i} I,
\end{aligned}
$$

where $\eta_{i}>2 L(N-1)\left\|P_{i}\right\|, P_{i}=\left(U_{1} T_{i} U_{1}^{T} E+U_{2} S_{i}\right), i=$ $2, \ldots, N$, and $L=\sum_{i=1}^{N} L_{i}$.
Suppose that we choose the average state of all node states as synchronized state; that is, $s(t)=(1 / N) \sum_{k=1}^{N} x_{k}(t)$. We have similar results. Before giving these results, we need some assumptions as follows:

Assumption $2^{\prime}$. Assume that there exist nonnegative constants $L_{i j}$ such that

$$
\begin{array}{r}
\left\|f\left(x_{i}(t), t\right)-f\left(x_{j}(t), t\right)\right\| \leq L_{i j}\left\|x_{i}(t)-x_{j}(t)\right\|, \\
i, j=1,2, \ldots, N .
\end{array}
$$

Assumption $3^{\prime}$. There exist matrices $P_{i}$ such that

$$
\begin{array}{r}
E^{T} P_{i}=P_{i}^{T} E \geq 0, \quad i=1,2, \ldots, N, \\
A^{T} P_{1}+P_{1}^{T} A<0, \\
\left(A+c \lambda_{i} \Gamma\right)^{T} P_{i}+P_{i}^{T}\left(A+c \lambda_{i} \Gamma\right) \leq-\varsigma_{i} I, \\
i=2, \ldots, N,
\end{array}
$$

where $\varsigma_{i}>(4 L / N)(N-1)\left\|P_{i}\right\|, L=\sum_{i=1}^{N} L_{i}$, and $L_{i}=$ $\sum_{k=1, k \neq i}^{N} L_{i k}$.

Theorem 13. Suppose that Assumptions 3, 2', and $3^{\prime}$ hold. By the controllers (17), the singular hybrid coupled network (1) can be synchronized to the average state of all node states in the finite time in the sense of Definition 2.

Corollary 14. Suppose that Assumptions 3 and $2^{\prime}$ hold, and matrix $E$ has the decomposition as (34) in Remark 11. By the controllers (17), if there exist matrices $T_{i} \in \mathbb{R}^{r \times r}, T_{i} \geq 0$, and $S_{i} \in \mathbb{R}^{(n-r) \times r}, i=1,2, \ldots, N$, such that

$$
\begin{gathered}
A^{T}\left(U_{1} T_{1} U_{1}^{T} E+U_{2} S_{1}\right)+\left(U_{1} T_{1} U_{1}^{T} E+U_{2} S_{1}\right)^{T} A<0, \\
\left(A+c \lambda_{i} \Gamma\right)^{T}\left(U_{1} T_{i} U_{1}^{T} E+U_{2} S_{i}\right) \\
\quad+\left(U_{1} T_{i} U_{1}^{T} E+U_{2} S_{i}\right)^{T}\left(A+c \lambda_{i} \Gamma\right) \leq-\eta_{i} I,
\end{gathered}
$$

where $\eta_{i}>(4 L / N)(N-1)\left\|P_{i}\right\|, P_{i}=\left(U_{1} T_{i} U_{1}^{T} E+U_{2} S_{i}\right)$, $i=2, \ldots, N, L=\sum_{i=1}^{N} L_{i}$, and $L_{i}=\sum_{k=1, k \neq i}^{N} L_{i k}$, the singular hybrid coupled network (1) can be synchronized to the average state of all node states in the finite time.

Remark 15. In this paper, we study finite-time synchronization of the singular hybrid coupled networks when the singular systems studied in this paper are assumed to be regular and impulse-free. However, it may be more complicated when we do not assume in advance that the systems are regular and impulse free. Synchronization or finite-time synchronization of singular coupled systems is worth discussing without the assumption that the considered systems are regular and impulsive free.

\section{An Illustrative Example}

In this section, a numerical example will be given to verify the theoretical results obtained earlier. 
Example 16. Consider the following singular hybrid coupled network which is similar to one given in [18]:

$$
E \dot{x}_{i}(t)=A x_{i}(t)+f\left(x_{i}(t), t\right)+c \sum_{j=1}^{6} b_{i j} \Gamma x_{j}(t)+u_{i},
$$

$$
i=1,2, \ldots, 6 \text {, }
$$

where $x_{i}(t)=\left(x_{i}^{1}(t), x_{i}^{2}(t)\right)^{T}, f\left(x_{i}(t), t\right)=((1 /$ 15) $\left.\tanh \left(x_{i}^{1}(t)\right), \tanh \left(x_{i}^{2}(t)\right)\right)^{T}, s(t)=(0,0)^{T}, L_{i}=1 / 15$, $L=2 / 5, c=1$, and

$$
\begin{gathered}
E=\left(\begin{array}{ll}
8 & 0 \\
0 & 0
\end{array}\right), \quad A=\left(\begin{array}{ccc}
-10 & 1 \\
1 & -10
\end{array}\right), \quad \Gamma=\left(\begin{array}{ll}
1 & 0 \\
0 & 1
\end{array}\right), \\
B=\left(\begin{array}{cccccc}
-5 & 1 & 1 & 1 & 1 & 1 \\
1 & -4 & 1 & 1 & 1 & 0 \\
1 & 1 & -4 & 1 & 0 & 1 \\
1 & 1 & 1 & -4 & 1 & 0 \\
1 & 1 & 0 & 1 & -4 & 1 \\
1 & 0 & 1 & 0 & 1 & -3
\end{array}\right) .
\end{gathered}
$$

Since $B$ is symmetric matrix and its six eigenvalues are $\lambda_{1}=$ $0, \lambda_{2}=-3, \lambda_{3}=-4, \lambda_{4}=-5, \lambda_{5}=-6$, and $\lambda_{6}=-6$, there exists a unitary matrix

$$
\begin{aligned}
W & =\left(W_{1}, \ldots, W_{6}\right) \\
& =\left(\begin{array}{cccccc}
-\frac{1}{\sqrt{6}} & 0 & 0 & 0 & -\frac{1}{\sqrt{6}} & -\frac{2}{\sqrt{6}} \\
-\frac{1}{\sqrt{6}} & \frac{1}{\sqrt{6}} & 0 & -\frac{1}{\sqrt{2}} & \frac{1}{\sqrt{6}} & 0 \\
-\frac{1}{\sqrt{6}} & 0 & -\frac{1}{\sqrt{2}} & 0 & -\frac{1}{\sqrt{6}} & \frac{1}{\sqrt{6}} \\
-\frac{1}{\sqrt{6}} & \frac{1}{\sqrt{6}} & 0 & \frac{1}{\sqrt{2}} & \frac{1}{\sqrt{6}} & 0 \\
-\frac{1}{\sqrt{6}} & 0 & \frac{1}{\sqrt{2}} & 0 & -\frac{1}{\sqrt{6}} & \frac{1}{\sqrt{6}} \\
-\frac{1}{\sqrt{6}} & -\frac{2}{\sqrt{6}} & 0 & 0 & \frac{1}{\sqrt{6}} & 0
\end{array}\right)
\end{aligned}
$$

such that $B=W \Lambda W^{T}$ and $\Lambda=\operatorname{diag}(0,-3,-4,-5,-6,-6)$.

Choose

$$
\begin{gathered}
M_{i}=\left(\begin{array}{cc}
1 & \frac{1}{10-\lambda_{i}} \\
0 & 1
\end{array}\right), \quad Q_{i}=\left(\begin{array}{cc}
\frac{1}{8} & 0 \\
\frac{1}{8\left(10-\lambda_{i}\right)} & \frac{1}{\lambda_{i}-10}
\end{array}\right), \\
P_{i}=\left(\begin{array}{ll}
1 & 0 \\
0 & 1
\end{array}\right),
\end{gathered}
$$

$\eta_{i}>4, i=1,2, \ldots, 6$, and $\beta=1 / 2$ satisfying Assumptions 3,4 , and 5. Under the controllers $u_{i}$ defined in Theorem 9, the singular hybrid system (39) can be synchronized in the finite time $t^{*}=7.1858$ according to Theorem 9 if $k=1$. If the controller gain $k=5, t^{*}=1.4372$. Corresponding simulation results are shown in Figures 1 and 2 with initial conditions $e(0)=\left(\begin{array}{ccccccc}1 & 3 & 2 & 0.7 & 2.5 & 0.3 \\ 0.5 & -1 & 0.8 & -2 & 1.5 & -0.5\end{array}\right)$.

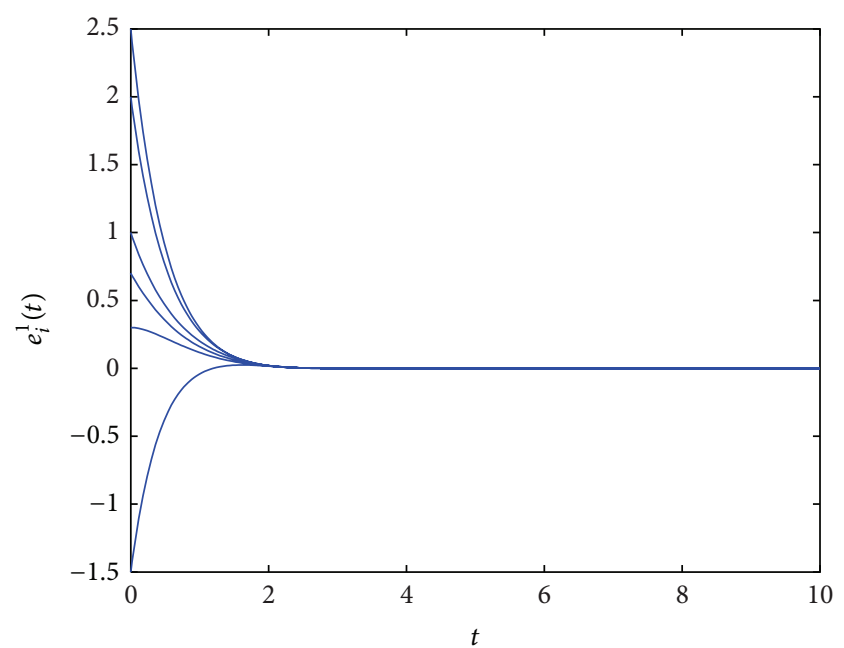

Figure 1: Error variable $e_{i}^{1}(t)(i=1,2, \ldots, 6)$ of system (39) with $k=1$.

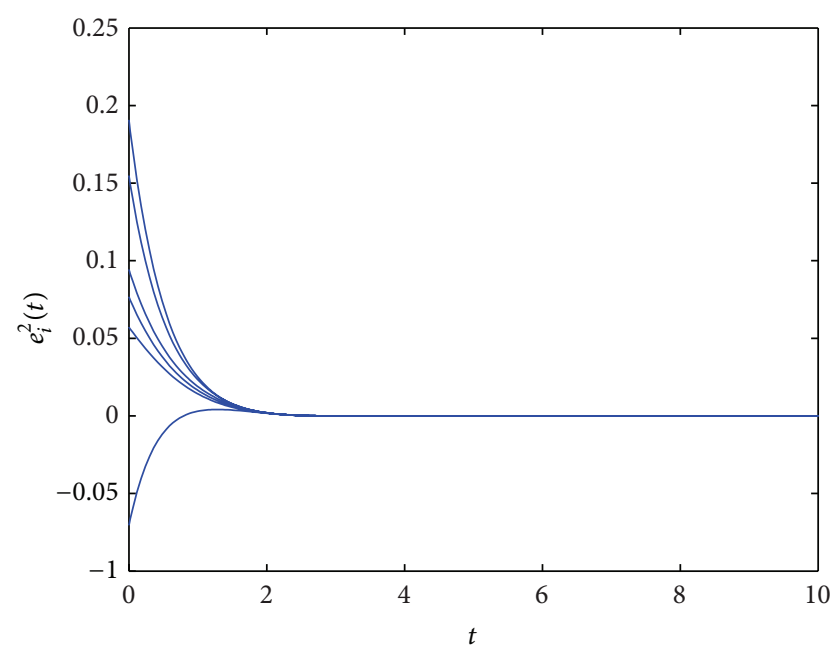

FIGURE 2: Error variable $e_{i}^{2}(t)(i=1,2, \ldots, 6)$ of system (39) with $k=1$.

\section{Conclusions}

In this paper, we discuss finite-time synchronization of the singular hybrid coupled networks with the assumption that the considered singular systems are regular and impulsivefree. Some sufficient conditions are derived to ensure finitetime synchronization of the singular hybrid coupled networks under a state feedback controller by finite-time stability theory. A numerical example is finally exploited to show the effectiveness of the obtained results. It will be an interesting topic for the future researches to extend new methods to study synchronization, robust control, pinning control, and finite-time synchronization of singular hybrid coupled networks without the assumption that the considered singular systems are regular and impulsive-free. 


\section{Acknowledgments}

This work was jointly supported by the National Natural Science Foundation of China under Grant nos. 61272530 and 11072059 and the Jiangsu Provincial Natural Science Foundation of China under Grant no. BK2012741.

\section{References}

[1] F. L. Lewis, "A survey of linear singular systems," Circuits, Systems, and Signal Processing, vol. 5, no. 1, pp. 3-36, 1986.

[2] S. Xu, P. Van Dooren, R. Ştefan, and J. Lam, "Robust stability and stabilization for singular systems with state delay and parameter uncertainty," IEEE Transactions on Automatic Control, vol. 47, no. 7, pp. 1122-1128, 2002.

[3] G. Lu and D. W. C. Ho, "Generalized quadratic stability for continuous-time singular systems with nonlinear perturbation," IEEE Transactions on Automatic Control, vol. 51, no. 5, pp. 818$823,2006$.

[4] G. Grammel, "Robustness of exponential stability to singular perturbations and delays," Systems \& Control Letters, vol. 57, no. 6, pp. 505-510, 2008.

[5] D. M. Stipanović and D. D. Šiljak, "Robust stability and stabilization of discrete-time non-linear systems: the LMI approach," International Journal of Control, vol. 74, no. 9, pp. 873-879, 2001.

[6] E. Uezato and M. Ikeda, "Strict LMI conditions for stability, robust stabilization, and $H_{\infty}$ control of descriptor systems," in Proceedings of the 38th IEEE Conference on Decision and Control, pp. 4092-4097, Phoenix, Ariz, USA, 1999.

[7] P. Wang and J. Zhang, "Stability of solutions for nonlinear singular systems with delay," Applied Mathematics Letters, vol. 25, no. 10, pp. 1291-1295, 2012.

[8] Y. Zhang, C. Liu, and X. Mu, "Robust finite-time stabilization of uncertain singular Markovian jump systems," Applied Mathematical Modelling, vol. 36, no. 10, pp. 5109-5121, 2012.

[9] L. Q. Zhang, B. Huang, and J. Lam, "LMI synthesis of $\mathrm{H}_{2}$ and mixed $\mathrm{H}_{2} / \mathrm{H}_{\mathrm{\infty}}$ controllers for singular systems," IEEE Transactions on Circuits and Systems II, vol. 50, pp. 615-626, 2003.

[10] I. Masubuchi, Y. Kamitane, A. Ohara, and N. Suda, " $H_{\infty}$ control for descriptor systems: a matrix inequalities approach," Automatica, vol. 33, no. 4, pp. 669-673, 1997.

[11] S. Xu and J. Lam, Robust Control and Filtering of Singular Systems, vol. 332 of Lecture Notes in Control and Information Sciences, Springer, Berlin, Germany, 2006.

[12] E. Fridman, " $H_{\infty}$-control of linear state-delay descriptor systems: an LMI approach," Linear Algebra and Its Applications, vol. 15, pp. 271-302, 2002.

[13] Y. Xia, J. Zhang, and E.-K. Boukas, "Control of discrete singular hybrid systems," Automatica, vol. 44, no. 10, pp. 2635-2641, 2008.

[14] S. Xu and C. Yang, " $H_{\infty}$ state feedback control for discrete singular systems," IEEE Transactions on Automatic Control, vol. 45, no. 7, pp. 1405-1409, 2000.

[15] M. S. Mahmoud and N. B. Almutairi, "Stability and implementable $H_{\infty}$ filters for singular systems with nonlinear perturbations," Nonlinear Dynamics, vol. 57, no. 3, pp. 401-410, 2009.

[16] Z. G. Feng and J. Lam, "Robust reliable dissipative filtering for discrete delay singular systems," Signal Processing, vol. 92, pp. 3010-3025, 2012.
[17] W. J. Xiong, D. W. C. Ho, and J. D. Cao, "Synchronization analysis of singular hybrid coupled networks," Physics Letters A, vol. 372, pp. 6633-6637, 2008.

[18] J. F. Zeng and J. D. Cao, "Synchronisation in singgular hybrid complex networks with delayed coupling," International Journal of Systems, Control and Communications, vol. 3, pp. 144-157, 2011.

[19] J. H. Koo, D. H. Ji, and S. C. Won, "Synchronization of singular complex dynamical networks with time-varying delays," Applied Mathematics and Computation, vol. 217, no. 8, pp. 39163923, 2010.

[20] H. J. Li, Z. J. Ning, Y. H. Yin, and Y. Tang, "Synchronization and state estimation for singular complex dynamical networks with time-varying delays," Communications in Nonlinear Science and Numerical Simulation, vol. 18, pp. 194-208, 2013.

[21] S. Li, W. Tang, and J. Zhang, "Robust control for synchronization of singular complex delayed networks with stochastic switched coupling," International Journal of Computer Mathematics, vol. 89, no. 10, pp. 1332-1344, 2012.

[22] J. Xu and J. Sun, "Finite-time stability of linear time-varying singular impulsive systems," IET Control Theory \& Applications, vol. 4, no. 10, pp. 2239-2244, 2010.

[23] X. Huang, W. Lin, and B. Yang, "Global finite-time stabilization of a class of uncertain nonlinear systems," Automatica, vol. 41, no. 5, pp. 881-888, 2005.

[24] L. Wang, S. Sun, and C. Xia, "Finite-time stability of multi-agent system in disturbed environment," Nonlinear Dynamics, vol. 67, no. 3, pp. 2009-2016, 2012.

[25] V. T. Haimo, "Finite time controllers," SIAM Journal on Control and Optimization, vol. 24, no. 4, pp. 760-770, 1986.

[26] S. P. Bhat and D. S. Bernstein, "Finite-time stability of continuous autonomous systems," SIAM Journal on Control and Optimization, vol. 38, no. 3, pp. 751-766, 2000.

[27] Y. Chen and J. H. Lü, "Finite time synchronization of complex dynamical networks," Journal of Systems Science and Mathematical Sciences, vol. 29, no. 10, pp. 1419-1430, 2009.

[28] H. Wang, Z. Han, Q. Xie, and W. Zhang, "Finite-time chaos control of unified chaotic systems with uncertain parameters," Nonlinear Dynamics, vol. 55, no. 4, pp. 323-328, 2009.

[29] Y. Z. Sun, W. Li, and D. H. Zhao, "Finite-time stochastic outer synchronization between two complex dynamical networks with different topologies," Chaos, vol. 22, no. 2, Article ID 023152, 2012.

[30] N. Cai, W. Li, and Y. Jing, "Finite-time generalized synchronization of chaotic systems with different order," Nonlinear Dynamics, vol. 64, no. 4, pp. 385-393, 2011.

[31] X. Yang and J. Cao, "Finite-time stochastic synchronization of complex networks," Applied Mathematical Modelling, vol. 34, no. 11, pp. 3631-3641, 2010.

[32] S. Li and Y.-P. Tian, "Finite time synchronization of chaotic systems," Chaos, Solitons and Fractals, vol. 15, no. 2, pp. 303-310, 2003.

[33] W. L. Yang, X. D. Xia, Y. C. Dong, and S. W. Zheng, "Finite time synchronization between two different chaotic systems with uncertain parameters," Computer \& Information Science, vol. 3, pp. 174-179, 2010.

[34] L. Wang and F. Xiao, "Finite-time consensus problems for networks of dynamic agents," IEEE Transactions on Automatic Control, vol. 55, no. 4, pp. 950-955, 2010.

[35] S. Yang, J. Cao, and J. Lu, "A new protocol for finite-time consensus of detail-balanced multi-agent networks," Chaos, vol. 22, Article ID 043134, 2012. 
[36] L. Wang, Z. Chen, Z. Liu, and Z. Yuan, "Finite time agreement protocol design of multi-agent systems with communication delays," Asian Journal of Control, vol. 11, no. 3, pp. 281-286, 2009.

[37] Q. Hui, W. M. Haddad, and S. P. Bhat, "Finite-time semistability and consensus for nonlinear dynamical networks," IEEE Transactions on Automatic Control, vol. 53, no. 8, pp. 1887-1900, 2008.

[38] Y. Shen and X. Xia, "Semi-global finite-time observers for nonlinear systems," Automatica, vol. 44, no. 12, pp. 3152-3156, 2008. 


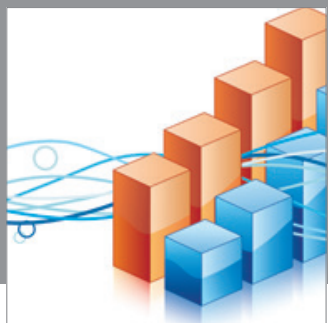

Advances in

Operations Research

mansans

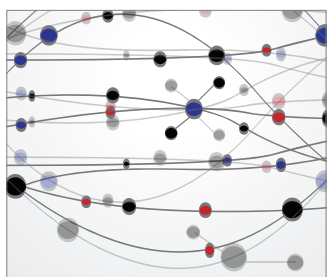

The Scientific World Journal
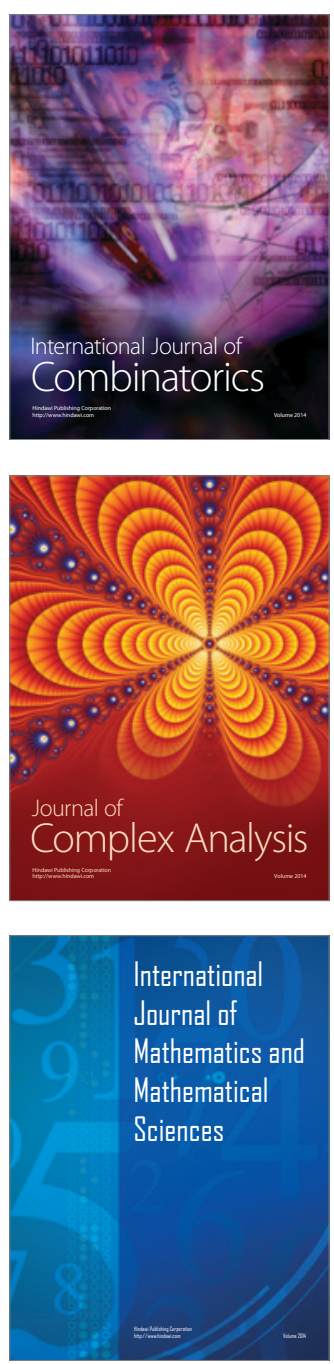
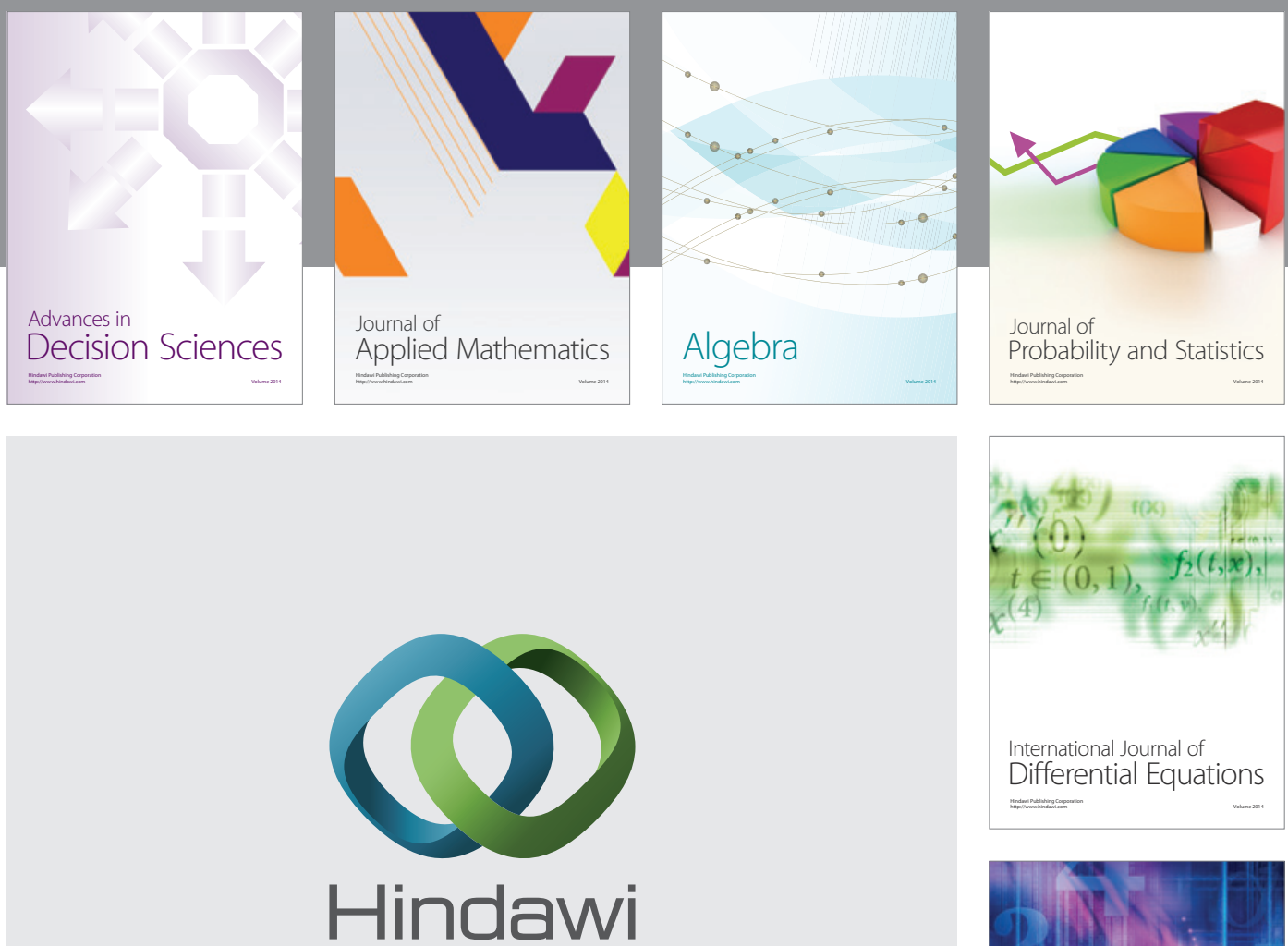

Submit your manuscripts at http://www.hindawi.com
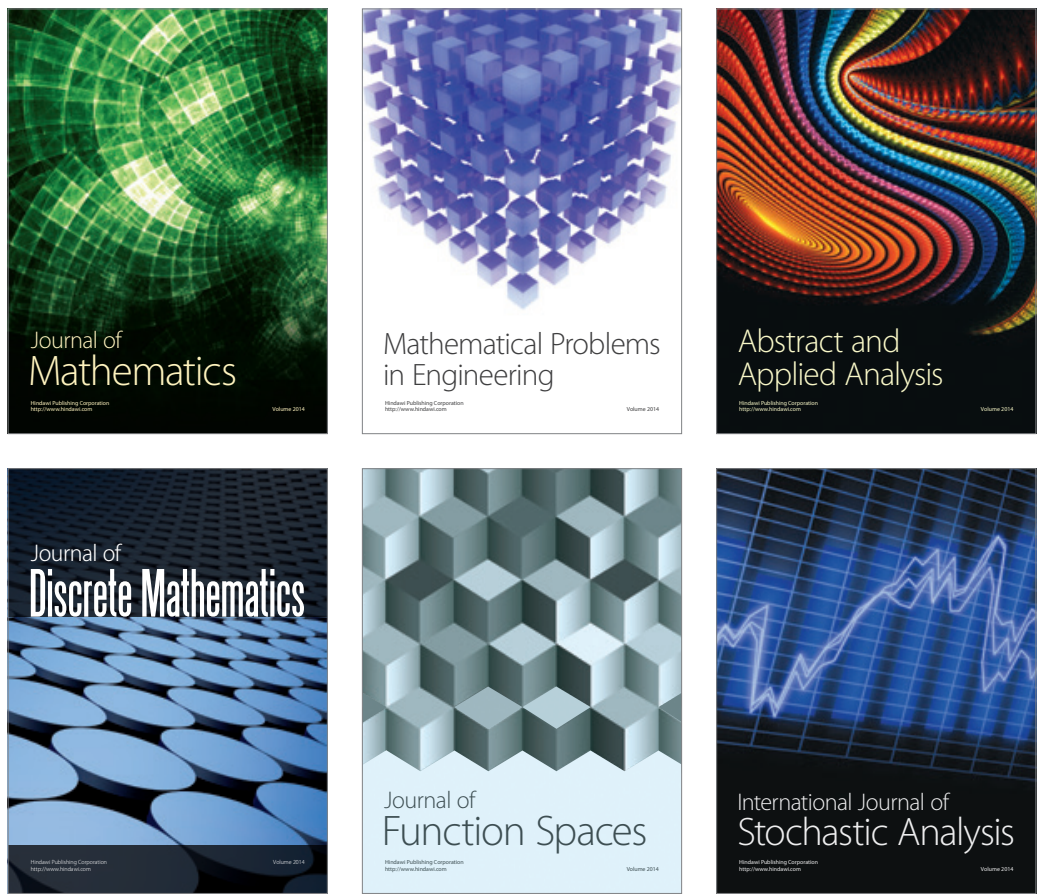

Journal of

Function Spaces

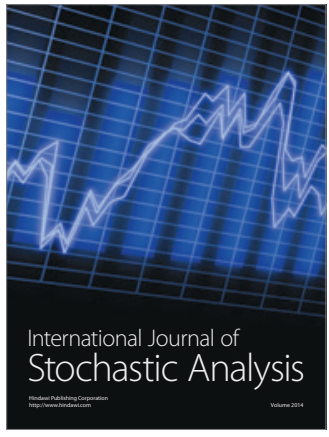

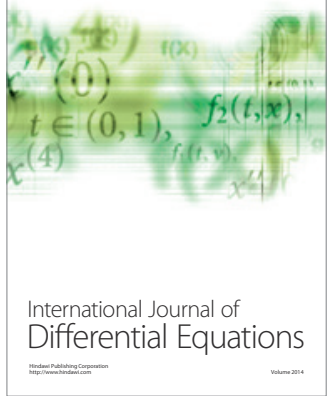
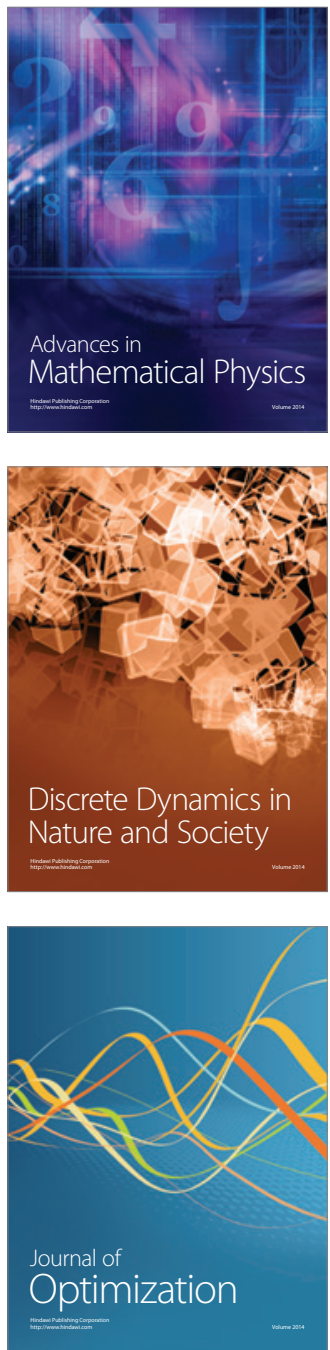